\title{
On the effects of suction and injection on the absolute instability of the rotating-disk boundary layer
}

R. J. Lingwood

Citation: Physics of Fluids 9, 1317 (1997); doi: 10.1063/1.869246

View online: https://doi.org/10.1063/1.869246

View Table of Contents: http://aip.scitation.org/toc/phf/9/5

Published by the American Institute of Physics

\section{Articles you may be interested in}

On the stability of von Kármán rotating-disk boundary layers with radial anisotropic surface roughness

Physics of Fluids 28, 014104 (2016); 10.1063/1.4939793

A new way to describe the transition characteristics of a rotating-disk boundary-layer flow

Physics of Fluids 24, 031701 (2012); 10.1063/1.3696020

Crossflow instability of finite Bödewadt flows: Transients and spiral waves

Physics of Fluids 21, 114107 (2009); 10.1063/1.3262817

Laminar-turbulent boundary-layer transition over a rough rotating disk

Physics of Fluids 15, 2441 (2003); 10.1063/1.1586916

An experimental study of boundary-layer transition over a rotating, compliant disk

Physics of Fluids 11, 3340 (1999); 10.1063/1.870193

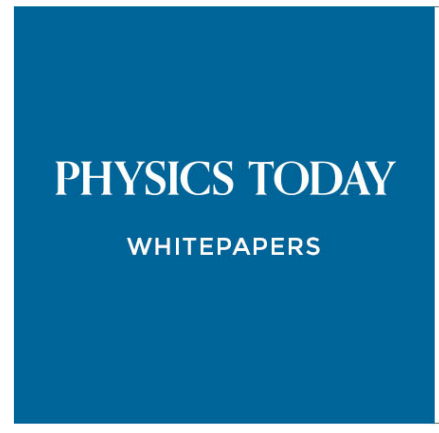




\title{
On the effects of suction and injection on the absolute instability of the rotating-disk boundary layer
}

\author{
R. J. Lingwood \\ Department of Engineering, University of Cambridge, Trumpington Street, Cambridge CB2 1PZ, \\ United Kingdom
}

(Received 4 November 1996; accepted 17 January 1997)

In this paper we are concerned with the theoretical behavior of the laminar von Kármán boundary-layer flow, extending the work presented by Lingwood [J. Fluid Mech. 299, 17 (1995); 314, 373 (1996)] to the flow with mass transfer at the surface of the disk. It is known that, within specific regions of the parameter space, the flow is absolutely unstable in the radial direction, i.e. disturbances grow in time at every radial location within these regions. Uniform suction through the disk is shown to delay the onset of absolute instability, while uniform injection promotes the onset. By comparing suction and injection velocities of the same magnitude, it is shown that suction has a greater stabilizing effect on the absolute instability than the destabilizing effect of injection. Suction is also strongly stabilizing to both stationary and travelling inviscidly unstable branch-1 modes; injection is destabilizing. Stationary viscously unstable branch-2 modes are strongly stabilized and destabilized by suction and injection, respectively, but travelling branch-2 modes are shown to be much less sensitive to mass transfer through the disk. (C) 1997 American Institute of Physics. [S1070-6631(97)03005-5]

\section{INTRODUCTION}

The von Kármán boundary layer (with and without mass transfer) has an inflectional mean velocity component and therefore, like swept-wing boundary layers (which have a similarly inflectional mean velocity component), is susceptible to inviscid crossflow instability. Crossflow instability was first noticed experimentally in the flow over a swept wing by Gray, ${ }^{1}$ where it manifested itself as a striped pattern fixed to the wing surface consisting of a series of stationary vortices in the boundary layer. Experimental evidence of crossflow instability of the von Kármán boundary layer is given by Gregory, Stuart and Walker. ${ }^{2}$ As well as the inviscidly unstable crossflow modes (termed branch 1 here), the von Kármán boundary layer has a second convectively unstable mode (branch 2) that was discovered by Malik ${ }^{3}$ and has been shown by $\mathrm{Hall}^{4}$ to be due to a balance between viscous and Coriolis forces. Branch-2 travelling waves can be convectively unstable to very low Reynolds numbers (the lowest critical Reynolds number is calculated here to be about 64.45). Therefore, these modes are usually observed in experiments as travelling (rather than stationary) disturbances at lower Reynolds numbers than the crossflow disturbances that dominate at high Reynolds numbers before transition; see, for example, Fedorov ${ }^{5}$ and Lingwood. $^{6}$ The recent study of the stability of the von Kármán boundary layer by Lingwood ${ }^{7}$ showed that, above a critical Reynolds number, the flow is radially absolutely unstable, i.e. the response to a transient disturbance grows with time at fixed radial positions. This instability is due to a coalescence of the inviscidly unstable mode and a third mode (branch 3 ) that is spatially damped and inwardly propagating. The absolute instability has been confirmed experimentally by Lingwood ${ }^{6}$ and it is suggested that, in the absence of possibly more dominant mechanisms such as secondary instability of the mean flow distorted by primary stationary crossflow vortices (e.g., Kohama ${ }^{8,9}$ and Balachandar, Streett and Malik ${ }^{10}$ ), the absolute instability mechanism is responsible for the onset of nonlinear behaviour and laminar-turbulent transition.

It was shown by von Kármán ${ }^{11}$ that there is an exact similarity solution of the Navier-Stokes equations for the steady incompressible flow due to an infinite rotating disk. As noted by Batchelor, ${ }^{12}$ the resulting ordinary differential equations are also applicable to the case with a uniform flow through the surface of the disk; both uniform suction and injection. The idea of using suction to maintain laminar flow to higher Reynolds numbers than would be possible without suction is well established. For example, the use of distributed suction has been shown experimentally (Pfenninger and Bacon $^{13}$ ) and theoretically (Hall, Malik and Poll ${ }^{14}$ ) to stabilize the attachment-line flow at the leading edge of swept wings. Away from the attachment line, suction of the threedimensional boundary layer on a swept wing should extend the laminar region by reducing the magnitude of the secondary (crossflow) velocity, by decreasing the boundary-layer thickness and by changing the secondary velocity profile to one that is inherently more stable. Similar ideas should be applicable to the rotating-disk boundary layer with suction, although it was shown by Stuart ${ }^{15}$ that the shape of the mean velocity profiles is not significantly affected by uniform suction. The effects of suction on the stationary convectively unstable (branch-1 and branch-2) modes in the rotating-disk boundary layer have been studied using linear stability theory by Dhanak. ${ }^{16}$ Suction was shown to increase the critical Reynolds numbers associated with these stationary branch-1 and branch- 2 modes, and to reduce the convectively unstable region of the wavenumber/Reynolds number parameter space. Conversely, injection was shown to decrease the critical Reynolds numbers and to expand the convectively unstable region of the wavenumber/Reynolds number parameter space. Further, Bassom and Seddougui ${ }^{17}$ have investigated the effects of suction on the nonlinear stability 
of stationary branch- 2 modes in the rotating-disk boundary layer at asymptotically large Reynolds number. They found that suction lowers the threshold amplitude for disturbance (disturbances smaller than the threshold decay as they develop away from the neutral position, but those bigger than the threshold grow explosively), while injection raises the threshold amplitude. Thus, from a linear viewpoint suction is stabilizing to stationary branch-2 waves but, since the threshold amplitude decreases with increasing suction, Bassom and Seddougui ${ }^{17}$ suggested that an experiment with suction would need less forcing than the zero-suction case for subcritical stationary instability to occur. Conversely, an experiment with fluid injection would need stronger forcing for subcritical instability, despite the linear destabilization of stationary branch-2 waves due to injection. Gregory and Walker ${ }^{18}$ performed experiments on a rotating disk with distributed suction and suction slots. These results will be discussed further in Sec. IV. Interest in mass injection into boundary-layer flows has been prompted by the wish to cool turbine blades and the surfaces of high speed aircraft. To model these problems properly, however, it would be necessary to take account of heat transfer effects as well as mass transfer through the porous surfaces. A recent paper by Seddougui and Bassom ${ }^{19}$ investigates the effects of both heat and mass transfer (and compressibility) on the rotating-disk boundary layer. This analysis is restricted to the convective instability of stationary branch-2 modes at an asymptotically large Reynolds number.

The main purpose of this paper is to assess the effect of mass transfer through the disk on the absolute instability mechanism that exists in the zero-suction case. However, the effects on the two convectively unstable branches of the dispersion relation are also discussed; emphasis is given to new results concerning travelling (rather than stationary) modes. As discussed by Huerre and Monkewitz, ${ }^{20}$ the response of the flow to impulsive forcing shows whether it is convectively or absolutely unstable. Following the work of Briggs ${ }^{21}$ and Bers ${ }^{22}$ in the field of plasma physics, absolute instability can be identified by singularities in the dispersion relationship that occur when modes associated with waves propagating in opposite directions coalesce. Such points have become known as pinch points. Although it is usual in linear stability analysis to choose either temporal or spatial theories, where absolute instability is suspected, it is necessary to perform a spatio-temporal analysis.

The structure of the paper is as follows. A brief statement of the problem is given in Sec. II, which includes discussion of the mean flow (Sec. II A) and the linear stability analysis (Sec. II B). Results of the analysis are presented in Sec. III, which includes a subsection on the effects of mass transfer on the convective instability of branches 1 and 2 (both stationary and travelling), and a subsection on the mass-transfer effects on the radial absolute instability involving branches 1 and 3 . These results are followed by a discussion in Sec. IV and concluding remarks in Sec. V.

\section{STATEMENT OF THE PROBLEM}

\section{A. The mean flow}

The disk is modelled as an infinite planar disk rotating at a constant angular frequency, $\Omega^{*}$, about the vertical axis that passes through the centre of the disk (asterisks indicate dimensional quantities). Cylindrical-polar coordinates are used, $r^{*}$ being the radial distance from the axis of rotation, $\theta$ the polar angle in the direction of rotation and $z^{*}$ the normal distance from the disk. The mean flow relative to the disk is given by von Kármán's ${ }^{11}$ exact similarity solution to the Navier-Stokes equations. The dimensionless similarity variables of the solution are defined by

$$
\begin{aligned}
& U(z)=\frac{U^{*}}{r^{*} \Omega^{*}}, \quad V(z)=\frac{V^{*}}{r^{*} \Omega^{*}}, \\
& W(z)=\frac{W^{*}}{\left(\nu^{*} \Omega^{*}\right)^{1 / 2}}, \quad P(z)=\frac{P^{*}}{\rho^{*} \nu^{*} \Omega^{*}},
\end{aligned}
$$

where $U, V, W$, are the non-dimensional radial, circumferential and axial base flow velocities in the rotating frame, respectively, $P$ is the pressure, $\rho^{*}$ and $\nu^{*}$ are the density and kinematic viscosity and $z=z^{*} / L^{*}$ is the non-dimensional axial coordinate, where $L^{*}=\left(\nu^{*} / \Omega^{*}\right)^{1 / 2}$ is the nondimensionalizing lengthscale.

The set of ordinary differential equations for $U, V, W$ and $P$ are given, for example, by Schlichting. ${ }^{23}$ As discussed by Stuart, ${ }^{15}$ because $W$ is independent of $r^{*}$, these equations can be applied to the case of uniform upwards or downwards flow, denoted by $W_{0}^{*}$, through the surface of the disk. The dimensional velocity $W_{0}^{*}$ is the constant value taken by $W^{*}$ at the surface, and the boundary conditions to the equations for the mean flow are

$$
\begin{aligned}
& U(0)=V(0)=0, \quad W(0)=\frac{W_{0}^{*}}{\left(\nu^{*} \Omega^{*}\right)^{1 / 2}} \equiv-a, \\
& P(0)=\text { const }=0, \\
& U(z \rightarrow \infty)=0, \quad V(z \rightarrow \infty)=-1,
\end{aligned}
$$

where $a$ is a positive constant for suction through the disk and is a negative constant for injection.

A double precision fourth-order Runge-Kutta integrator and a Newton-Raphson searching method were used to solve for $U, V, W$ and $P$. Figure 1 shows these quantities plotted against $z$ for a range of the parameter $a$. (Figure 1 is essentially the same as Fig. 1 of Dhanak, ${ }^{16}$ but here the profiles are given for a larger range of $a$ and the mean pressure profile is given as well as the velocity profiles.) In the radial direction, and directions close to the radial, the velocity profiles are inflectional. Table I gives some values of $U^{\prime}(0)$ and $V^{\prime}(0)$ (where the primes denote differentiation with respect to $z$ ) required to calculate the mean profiles. Values of $W(z \rightarrow \infty)$ and $P(z \rightarrow \infty)$ are also tabulated. When suction is applied the magnitude of the radial velocity is decreased and therefore the three-dimensionality of the boundary layer is reduced, while the magnitude of the axial flow at infinity is increased. Conversely, injection increases the magnitude of the radial flow and decreases the axial flow at infinity. The decrease and increase in the maximum radial velocity with 

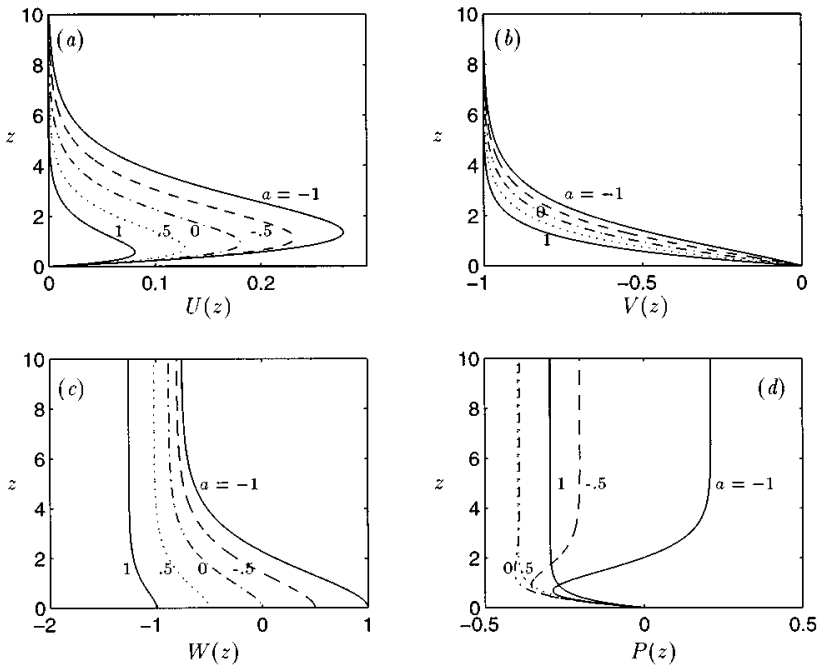

FIG. 1. Mean profiles for the rotating-disk flow for particular degrees of suction and injection: (a) radial velocity profiles, (b) circumferential velocity profiles, (c) axial velocity profiles, (d) pressure profiles.

suction and injection, respectively, are of almost equal magnitude (about $54 \%$ for $a= \pm 1$ compared with $a=0$ ). Figure 1 (c) indicates that the change in $W$ between $z=0$ and $z \rightarrow \infty$, which is quantified by $W(z \rightarrow \infty)+a$, tends to zero as $a \rightarrow \infty$. Further, $|W(z \rightarrow \infty)+a|$ tends to infinity as $a \rightarrow-\infty$. The variation in the mean pressure through the boundary layer increases as $a$ decreases.

Figure 2 shows the variation in the non-dimensional displacement thickness $\delta=\delta^{*} / L^{*}$, which is defined in terms of the azimuthal mean flow,

$$
\delta^{*}=\int_{0}^{\infty} 1+\frac{V^{*}}{\Omega^{*} r^{*}} d z^{*}=L^{*} \int_{0}^{\infty}(1+V) d z
$$

The non-dimensional momentum thickness $\bar{\theta}$ (not to be confused with the polar angle $\theta$ ) and the shape factor $H=\delta / \bar{\theta}$ are also given in Fig. 2, where

$$
\bar{\theta}=\int_{0}^{\infty} V(1+V) d z .
$$

The variation in $\delta$ with $a$ is shown to be linearly decreasing. The values of $\bar{\theta}$ and $H$ also decrease with increasing $a$. Thus, as suggested by Figs. 1(a) and (b), the effect of suction is to thin the boundary layer; the effect of injection is to thicken

TABLE I. Boundary conditions for the mean flow.

\begin{tabular}{rcclr}
\hline \hline$a$ & $U^{\prime}(0)$ & $V^{\prime}(0)$ & $W(z \rightarrow \infty)$ & $P(z \rightarrow \infty)$ \\
\hline-1.0 & 0.48948 & -0.30217 & -0.76071 & 0.21066 \\
-0.5 & 0.51457 & -0.43643 & -0.80721 & -0.20079 \\
0.0 & 0.51023 & -0.61592 & -0.88447 & -0.39115 \\
0.5 & 0.46688 & -0.85519 & -1.0213 & -0.39652 \\
1.0 & 0.38957 & -1.1752 & -1.2606 & -0.29450 \\
2.0 & 0.24242 & -2.0385 & -2.0577 & -0.11711 \\
3.0 & 0.16558 & -3.0121 & -3.0182 & -0.054791 \\
4.0 & 0.12474 & -4.0051 & -4.0078 & -0.031111 \\
5.0 & 0.099914 & -5.0027 & -5.0040 & -0.019963 \\
\hline \hline
\end{tabular}

Phys. Fluids, Vol. 9, No. 5, May 1997

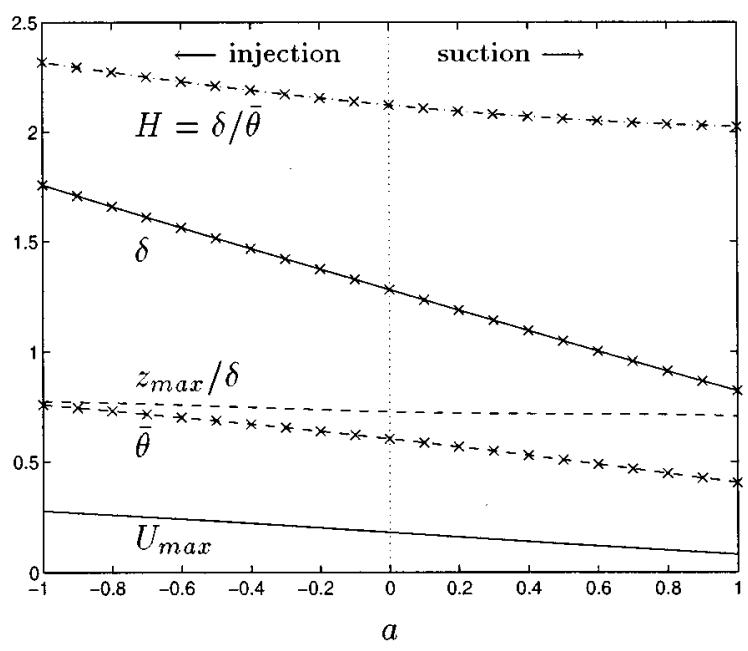

FIG. 2. Variation in the displacement and momentum thicknesses, the shape factor, $U_{\max }$ and $z_{\max } / \delta$ with $a$.

the boundary layer. Stuart ${ }^{15}$ showed that when the mean radial and azimuthal velocity profiles are plotted against $z / \delta$ for varying degrees of suction, the maxima in $U \equiv U_{\max }$ occur at almost constant $z / \delta$ and that for both $U$ and $V$ the variation with $a$ is predominantly one of scale rather than shape. In fact, Stuart notes that for the asymptotic suction profile $(a=\infty) H=2$, therefore the divergence of the plot of the shape factor (in Fig. 2) from 2 is a measure of the difference in shape of the profile from the asymptotic one. In Fig. $2, z / \delta$ at $U_{\max }$ (denoted by $z_{\max } / \delta$ ) is plotted against $a$ for both suction and injection. The constant trend shown by Stuart for the suction profiles is continued into negative $a$. As suggested by Fig. 1, the variation in $U_{\max }$ is almost linear in $a$.

\section{B. The linear stability analysis}

The stability analysis, applied at a radius $r_{a}^{*}$, involves imposing infinitesimally small disturbances on the mean flow. The local Reynolds number is $R=r_{a}^{*} \Omega^{*} L^{*} / \nu^{*}$ $=r_{a}^{*} / L^{*}=r_{a}$, and the non-dimensionalizing velocity, pressure and time-scales are $r_{a}^{*} \Omega^{*}, \quad \rho^{*} r_{a}^{* 2} \Omega^{* 2}$ and $L^{*} /\left(\Omega^{*} r_{a}^{*}\right)$, respectively. The instantaneous nondimensional velocities and pressure are given by

$$
\begin{aligned}
& \bar{u}(r, \theta, t, z)=\frac{r}{R} U(z)+u(r, \theta, t, z), \\
& \bar{v}(r, \theta, t, z)=\frac{r}{R} V(z)+v(r, \theta, t, z), \\
& \bar{w}(r, \theta, t, z)=\frac{1}{R} W(z)+w(r, \theta, t, z), \\
& \bar{p}(r, \theta, t, z)=\frac{1}{R^{2}} P(z)+p(r, \theta, t, z),
\end{aligned}
$$

where $u, v, w$ and $p$ are small perturbation quantities. 
The dimensionless Navier-Stokes equations are linearized with respect to the perturbation quantities. To make the linearized perturbation equations separable in $r, \theta$ and $t$, it is necessary to ignore variations in the Reynolds number with radius. This involves replacing the variable $r$, which appears in coefficients of the linearized equations, by $R$. Despite the fact that $\delta^{*}$ is constant (in $r^{*}$ ) for constant $a$, this sort of approximation is called the parallel-flow approximation. The terminology comes from analyses of growing boundary layers, such as the Blasius boundary layer, where variations in Reynolds number in the streamwise direction, due to growth of the boundary layer, are often ignored. Terms $O\left(R^{-2}\right)$ are neglected and the perturbation quantities are assumed to have normal-mode form, for example

$$
u=\hat{u}(z ; \alpha, \beta, \omega ; R) e^{i(\alpha r+\beta \theta-\omega t)},
$$

where $\hat{u}$ is the spectral representation of the radial perturbation velocity, $\alpha$ and $\beta=\bar{\beta} R$ are the radial and azimuthal wavenumbers, respectively, and $\omega$ is the frequency of the disturbance in the rotating frame. Because of the circumferential periodicity of this problem, $\beta$ takes integer values. Similar equations to (1) define $\hat{v}, \hat{w}$ and $\hat{p}$. Using certain transformations of the fundamental perturbation quantities, the perturbation equations may be written as a set of six first-order ordinary differential equations that include viscous, streamline-curvature and Coriolis effects; see Malik, Wilkinson and Orszag ${ }^{24}$ or Lingwood. ${ }^{7}$

To distinguish between a convectively and an absolutely unstable response, the governing equations are solved subject to an impulsive azimuthal line forcing, with prescribed integer $\beta$, such that the vertical velocity at $z=0$ is given by

$$
w(0 ; r, \theta, t)=\delta\left(r-r_{a}\right) \delta(t) e^{i \beta \theta},
$$

where $\delta\left(r-r_{a}\right)$ and $\delta(t)$ are the Dirac delta functions at $r_{a}$ and $t=0$, respectively. The response to point forcing can be obtained by summing over all integer values of $\beta$. The additional boundary conditions at $z=0$, given by the no-slip condition, are

$$
u(0 ; r, \theta, t)=v(0 ; r, \theta, t)=0,
$$

and as $z \rightarrow \infty$ it is required that all perturbations decay.

The solution of an inhomogeneous system such as this is described by Lingwood. ${ }^{25}$ The problem reduces to solving a Green's function of the form

$$
\begin{aligned}
w(z ; r, \theta, t)= & \frac{e^{i \beta \theta}}{(2 \pi)^{2}} \int_{F} \int_{A} \frac{\Phi(z ; \alpha, \omega ; \beta, R)}{\Delta_{0}(\alpha, \omega ; \beta, R)} \\
& \times e^{i\left(\alpha\left(r-r_{a}\right)-\omega t\right)} d \alpha d \omega,
\end{aligned}
$$

where $\Phi$ is a function of $z$ formed from a combination of the independent solution vectors of the governing ordinary differential equations, $\Delta_{0}=0$ is the dispersion relation, which is satisfied by the discrete eigenvalues of the homogeneous problem (the unforced case), and $A$ and $F$ are inversion contours in the $\alpha$ - and $\omega$-planes, respectively. The discrete eigenvalues provide a mapping between the $\alpha$ - and $\omega$-planes; the eigenvalues trace out paths in the $\alpha$-plane ( $\omega$-plane) as $\omega(\alpha)$ is varied. Trajectories in the $\alpha$-plane ( $\omega$-plane) given by a predetermined $\omega$-distribution ( $\alpha$-distribution), which could be complex, will be referred to as spatial (temporal) branches of the dispersion relation.

Briggs'21 method is used to calculate the timeasymptotic discrete solution, due to the discrete poles of (2) (i.e., zeros of the dispersion relation). Details of this method are given in Lingwood, ${ }^{25}$ where it is shown that the discrete response is sufficient to determine the nature of the instability. The discrete response to (2) neglects the continuous contributions from branch cuts in the $\alpha$-plane, which give the $z$-structure of the response close to the source of the initial perturbation and are caused by the complex square-roots taken to satisfy the boundary conditions as $z \rightarrow \infty$.

For fixed $\beta$, the Briggs' criterion for absolute instability requires a branch-point singularity between two, or more, spatial branches of the dispersion relation, of which at least two must lie in distinct halves of the $\alpha$-planes when $\omega_{i}$ is sufficiently large and positive. Such singularities have become known as pinch points because inherent in Briggs' method is the use of analytic continuation to deflect the inversion contours, and at these singularities the $A$-contour becomes pinched between the coalescing spatial branches. The pinching frequency $\omega^{o}$ is a branch point of the function $\alpha(\omega ; \beta, R)$ or, equivalently, $\alpha$ at the pinch point, $\alpha^{o}$, is a saddle point of the function $\omega^{o}(\alpha ; \beta, R)$ and at this point $\partial \omega / \partial \alpha=0$, although this condition is not sufficient for such points. If $\omega_{i}>0$ at the pinch point the flow is absolutely unstable, otherwise the flow is only convectively unstable or stable. A branch-point singularity between two spatial branches that originate in the same half of the $\alpha$-plane for large positive $\omega_{i}$ does not constitute a pinch point (even though $\partial \omega / \partial \alpha=0$ at such a point) and does not cause an absolute instability. This sort of branch point results in a second-order pole, which leads to transient algebraic growth that can be important if the second-order pole is near neutral but ultimately behaves exponentially as dictated by the sign of $\alpha_{i}$. This case will not be pursued here, but further details can be found in $\mathrm{Koch}^{26}$ and Henningson and Schmid. ${ }^{27}$

\section{RESULTS OF THE LINEAR STABILITY ANALYSIS}

\section{A. Convective instability of branches 1 and 2}

Neutral-stability curves for stationary $(\omega=0)$ waves are presented in Fig. 3 for a range of $a$. For the purposes of plotting Fig. 3(a), $\beta$ is treated as being continuous rather than taking only integer values. Essentially this figure is the same as Fig. 2 presented by Dhanak, ${ }^{16}$ although Dhanak considers just one case of mass injection $(a=-0.1)$, preferring to concentrate on the effects of suction on stationary modes. A comparison between Fig. 3 and Dhanak's results shows that the two analyses give qualitatively similar variations in the convective instability of stationary branch-1 and branch-2 modes with mass transfer. Suction increases the critical Reynolds numbers for convective instability of both branch 1 (i.e., the upper lobe in $a-c$ and the lower lobe in d) and branch 2 (i.e., the lower lobe in $\mathrm{a}-\mathrm{c}$ and the upper lobe in d); injection decreases the critical Reynolds numbers. Both here and in Dhanak, ${ }^{16}$ the wave angle, $\varepsilon=\tan ^{-1}\left(\bar{\beta} / \alpha_{r}\right)$, at the critical Reynolds numbers decreases 

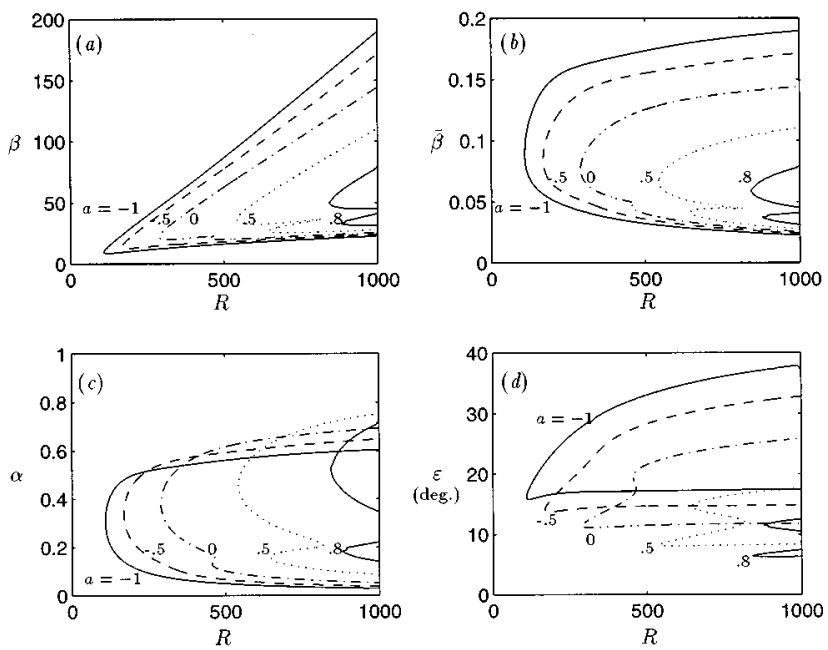

FIG. 3. Neutral-stability curves for stationary $(\omega=0)$ waves and particular degrees of suction and injection. The convectively unstable regions lie within the curves.

with increasing $a$. However, the solution method used by Dhanak is different to that used here and, as mentioned by Dhanak, in comparison with Malik $^{3}$ his method introduces some differences in the stability characteristics in the vicinity of the critical Reynolds numbers. Dhanak's stability analysis is based on the vorticity equation and the parallel-flow approximation is made at a different stage of the formulation. The formulation adopted in this paper is similar to Malik's, ${ }^{3}$ therefore it is unsurprising that, where comparisons can be made, the eigenvalues calculated here agree with those of Malik and not Dhanak. For $a=0.5$, the differences between the critical Reynolds numbers calculated here and by Dhanak are about $2 \%$ for branch 1 and about $8 \%$ for branch 2 .

Convective instability of travelling waves is also important in the rotating-disk boundary layer. Travelling waves are excited by freestream turbulence and have been observed experimentally, e.g. by Fedorov ${ }^{5}$ and Lingwood. ${ }^{6}$ In very close agreement with Balakumar and Malik, ${ }^{28}$ it has been calculated here that the lowest critical Reynolds number for convective instability for $a=0$ occurs for a travelling branch-2 mode at $\omega_{r} R \approx 7.8883128$. Disturbances have dimensional frequency $\omega^{*}=\omega R \Omega^{*}$, so the non-dimensional quantity $\omega_{r} R$ remains constant as disturbances propagate. In Fig. 4, neutral-stability curves are given for $\omega_{r} R=7.8883128$ and $a=-0.5,0,0.5$. As for the stationary waves, suction significantly increases the critical Reynolds number for convective instability of these travelling branch-1 modes; injection decreases it. However, the critical Reynolds number for branch 2 is far less sensitive to $a$ than branch 1 , although suction is still slightly stabilizing and injection is slightly destabilizing. Table II gives the critical parameters
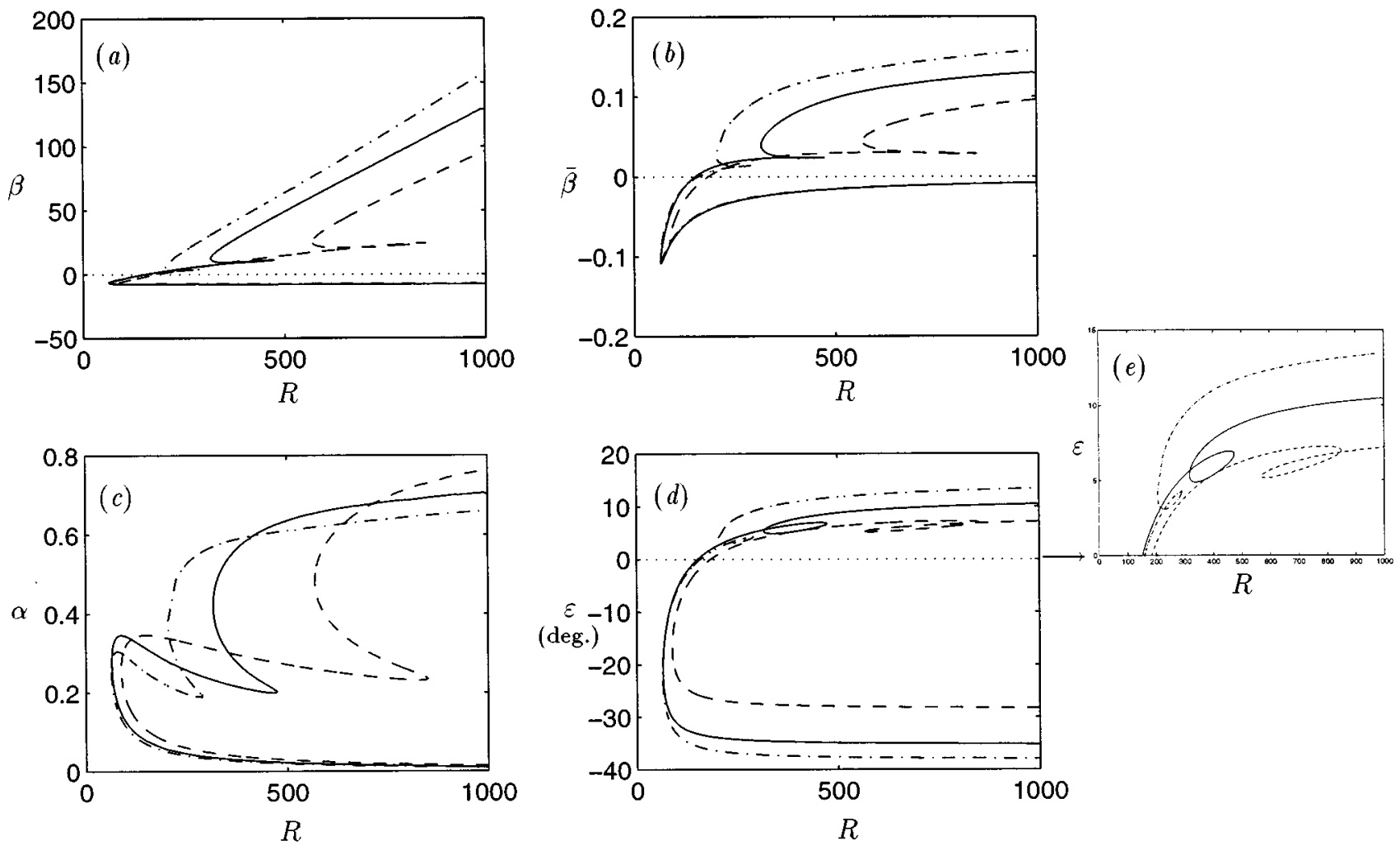

FIG. 4. Neutral-stability curves for travelling waves with $\omega_{r} R=7.8883128$, i.e. with the frequency that gives the lowest critical Reynolds number for $a=0$ : — - denotes $a=-0.5$, denotes $a=0$ and --- denotes $a=0.5$. The convectively unstable regions lie within the curves and (e) is an expanded view of $(d)$. 
TABLE II. Critical values for convective instability of branch 2 and $\omega_{r} R=7.8883128$.

\begin{tabular}{rcccc}
\hline \hline \multicolumn{1}{c}{$a$} & $R_{2}$ & $\bar{\beta}_{2}$ & $\alpha_{2}$ & $\varepsilon_{2}$ \\
\hline-0.5 & 62.9 & -0.102 & 0.264 & $-21.19^{\circ}$ \\
0.0 & 64.4 & -0.106 & 0.276 & $-21.07^{\circ}$ \\
0.5 & 87.9 & -0.0775 & 0.265 & $-16.29^{\circ}$ \\
\hline \hline
\end{tabular}

for branch 2 and $\omega_{r} R=7.8883128$ (denoted by the subscript 2).

Eigenfunctions of the radial, circumferential and axial velocity perturbations are given in Fig. 5. The parameters for Figs. 5(a)-(c) were chosen to characterize travelling branch-1 modes. That is, for each value of $a$, the eigenfunctions are given at the critical Reynolds number for convective instability of branch 1 at $\omega_{r} R=7.8883128$. The eigenfunctions for $a=-0.5$ are given at $R=223$, for $a=0$ they are given at $R=317$, and for $a=0.5$ they are given at $R=569$. The parameters for Figs. 5(d)-(f) were chosen to characterize travelling branch-2 modes. Because of the insensitivity of branch 2 to $a$, all these eigenfunctions are given for $R=100$ and $\beta=-5$. The eigenfunctions in Figs. $5(\mathrm{a})-(\mathrm{c})$ are normalized by the maximum of $\hat{v}$ (given in $\mathrm{b}$ ) for $a=0$; similarly the eigenfunctions in Figs. 5(d)-(f) are normalized by the maximum of $\hat{v}$ (given in e) for $a=0$. First considering the branch-1 eigenfunctions, for $a=0$ the maxima in $\hat{u}$ and $\hat{w}$ are about $16 \%$ and $8 \%$, respectively, of the maximum in $\hat{v}$. With injection of $a=-0.5$, the maximum magnitudes of $\hat{u}$ and $\hat{w}$ relative to $\hat{v}$ are increased to about $21 \%$ and $10 \%$, respectively. Whereas with suction of $a=0.5$, the maximum magnitudes of $\hat{u}$ and $\hat{w}$ relative to $\hat{v}$ are decreased to about $10 \%$ and $4 \%$, respectively. For the branch-1 eigenfunctions, $\hat{v}$ is almost constant for $z>6$, although suction seems to decrease the vertical extent of the variation in this eigenfunction slightly. The extent of both $\hat{u}$ and $\hat{w}$ is significantly greater. For branch 2 [in Figs. 5(d)(f), where $R=100$ ], the relative extents of $\hat{u}, \hat{v}$ and $\hat{w}$ are similar to those of the branch-1 eigenfunctions. The higher maxima of $\hat{u}$ are perhaps more pronounced. Suction shifts both maxima of $\hat{u}$ to lower $z$, while injection has the reverse effect. The maxima of $\hat{u}$ and $\hat{w}$ relative to $\hat{v}$ for $a=0$ are about $37 \%$ and $11 \%$, respectively. For $a=-0.5$ these values are increased to about $45 \%$ and $12 \%$, respectively, and decreased to about $29 \%$ and $9 \%$, respectively, for $a=0.5$. However, mass transfer does not have a marked effect on the general form of the branch-1 and branch-2 eigenfunctions. Note that it is only meaningful to compare the relative magnitudes of the eigenfunctions $(\hat{u}, \hat{v}$ or $\hat{w})$ for a given eigenvalue; the magnitude of, say, $\hat{u}(a=0)$ relative to $\hat{u}(a=0.5)$ is arbitrary.

\section{B. Absolute instability involving branches 1 and 3}

It has been established by Lingwood ${ }^{7}$ that a linear stability analysis predicts that the zero- $a$ case is radially absolutely unstable beyond a critical Reynolds number. Figure 6 compares marginal curves for absolute instability $\left(\omega_{i}^{o}=0\right)$ for $a= \pm 1$ with $a=0$. For suction with $a=1$ the critical Reynolds number for the onset of absolute instability is increased by a factor of nearly 3.7 compared with the zerosuction case. Thus, suction has a significant stabilizing effect on the absolute instability mechanism. Conversely, injection with $a=-1$ reduces the critical Reynolds number to about $40 \%$ of its value for $a=0$. The criteria for the marginal curves for absolute instability are that $\partial \omega / \partial \alpha=0$, i.e. there is a branch point $\omega^{o}$ of $\alpha\left(\omega^{o} ; \beta, R\right) \equiv \alpha^{o}$, that the branch point is of the pinch-type and that $\omega_{i}^{o}=0\left(\omega_{i}^{o}>0\right.$ implies absolute instability). In Fig. 6 for $a=0$ and $a=-1$, two trajectories of branch points with $\omega_{i}^{o}=0$ are given: $A$ and $B$. All the branch points on the $A$-trajectories, for both $a=0$ and $a=-1$, are of the pinch type. However, the branch points on the $B$-trajectories change from being non-pinching to pinching with increasing Reynolds number. The $B$-trajectories end at low Reynolds numbers where it was no longer possible to find marginal $\left(\omega_{i}^{o}=0\right)$ branch points. Figure 7 shows $\alpha^{o}$ in the complex $\alpha$-plane at the critical Reynolds number for the onset of absolute instability for a range of $a$. The solid lines in this figure indicate the separation of the two spatial branches involved in each branch point as $\omega_{i} \rightarrow \infty$ away from $\omega_{i}^{o}$. Since in each case the two spatial branches separate into the distinct half planes, all these branch points are of the pinch type and qualify as defining points on the marginal curves of absolute instability. Figure 8 shows a similar type of graph but for a selection of branch points on both trajectories $A$ and $B$ from Fig. 6 for $a=-1$. The separation of the two spatial branches into the distinct halves of the complex $\alpha$-plane as $\omega_{i} \rightarrow \infty$ defines the trajectory- $A$ branch points as pinch points. For $R=1000$ and $R=3000$ on trajectory $B$, the branch points are also pinching but for lower Reynolds numbers both spatial branches separate into the upper-half $\alpha$-plane, which means that these branch points are nonpinching and cannot constitute an absolute instability. These non-pinching branch points are a coalescence of the two (outwardly) convectively unstable modes: branches 1 and 2 . The change in character of the branch points on trajectory $B$ is due to the fact that between $R=900$ and $R=1000$ on trajectory $B$, branches 1,2 and 3 (branch 3 is the spatiallydamped inwardly propagating mode that exists in the lowerhalf $\alpha$-plane for $\omega_{i} \rightarrow \infty$ ) all coalesce and at that point the marginal branch point switches from involving only branches 1 and 2 to involving branch 3 . So, returning to Fig. 6 , there are marginal pinch points on the $A$-trajectories and above certain Reynolds numbers there are also marginal pinch points on the $B$-trajectories. In general, when there are several pinch points for a particular Reynolds number and $\beta$, the discrete time-asymptotic response is dominated by the pinch point that lies highest in the complex $\omega$-plane. This implies that for fixed $\beta$ with marginal pinch points on both trajectories $A$ and $B$, the most critical pinch point is the one lying on the trajectory at the lower Reynolds number; the other pinch point then has $\omega_{i}^{o}<0$ for that particular $R$ and $\beta$ and is subcritical. To define limiting curves of absolute instability in this way for $a=-1$, it is necessary to jump from marginal pinch points on trajectory $A$ to those on tra- 

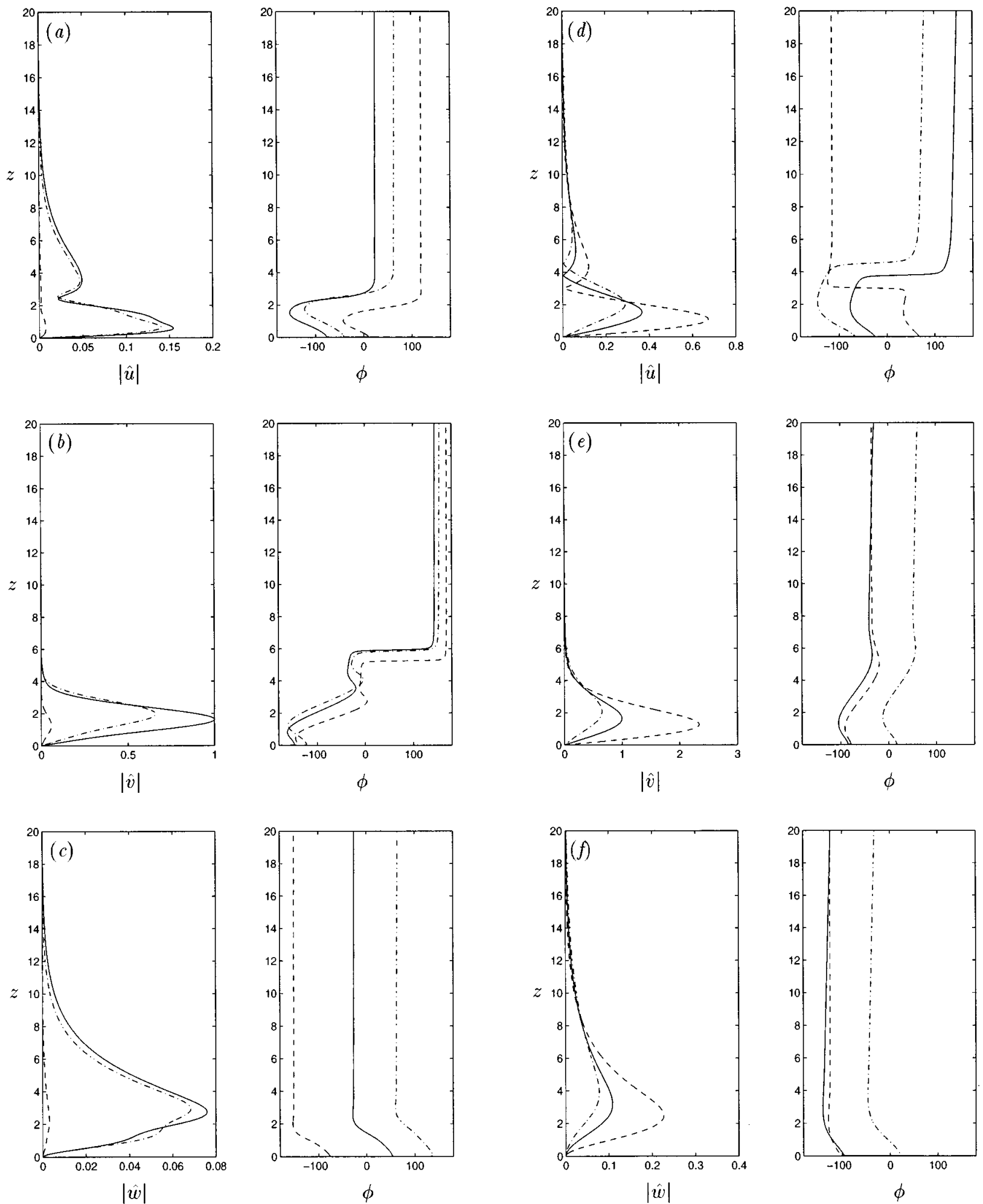

FIG. 5. Magnitude and phase $\phi$ of the eigenfunctions, normalized by the maximum of $\hat{v}$ for $a=0$ : (a) - (c) at the critical parameters for the onset of convective instability for $\omega_{r} R=7.8883128$ on branch 1 , (d)-(f) at $R=100, \beta=-5$ and $\omega_{r} R=7.8883128$, i.e. for branch 2 . (a) and (d) radial perturbation velocity. (b) and (e) circumferential perturbation velocity. (c) and (f) axial perturbation velocity. The phase is given from $-180^{\circ}$ to $180^{\circ}$ and $-\cdot-\cdot$ denotes $a=-0.5,-$ denotes $a=0$ and --- denotes $a=0.5$.

jectory $B$, which introduces discontinuities in certain planes in Fig. 6. Below $R=2500$, it is not necessary to jump trajectories for $a=0$.

Despite the complications of defining the marginal curves for absolute instability at high Reynolds numbers, the critical Reynolds numbers for the onset are defined unambiguously. Note also that as $a$ decreases stationary waves become absolutely unstable at Reynolds numbers increas- 

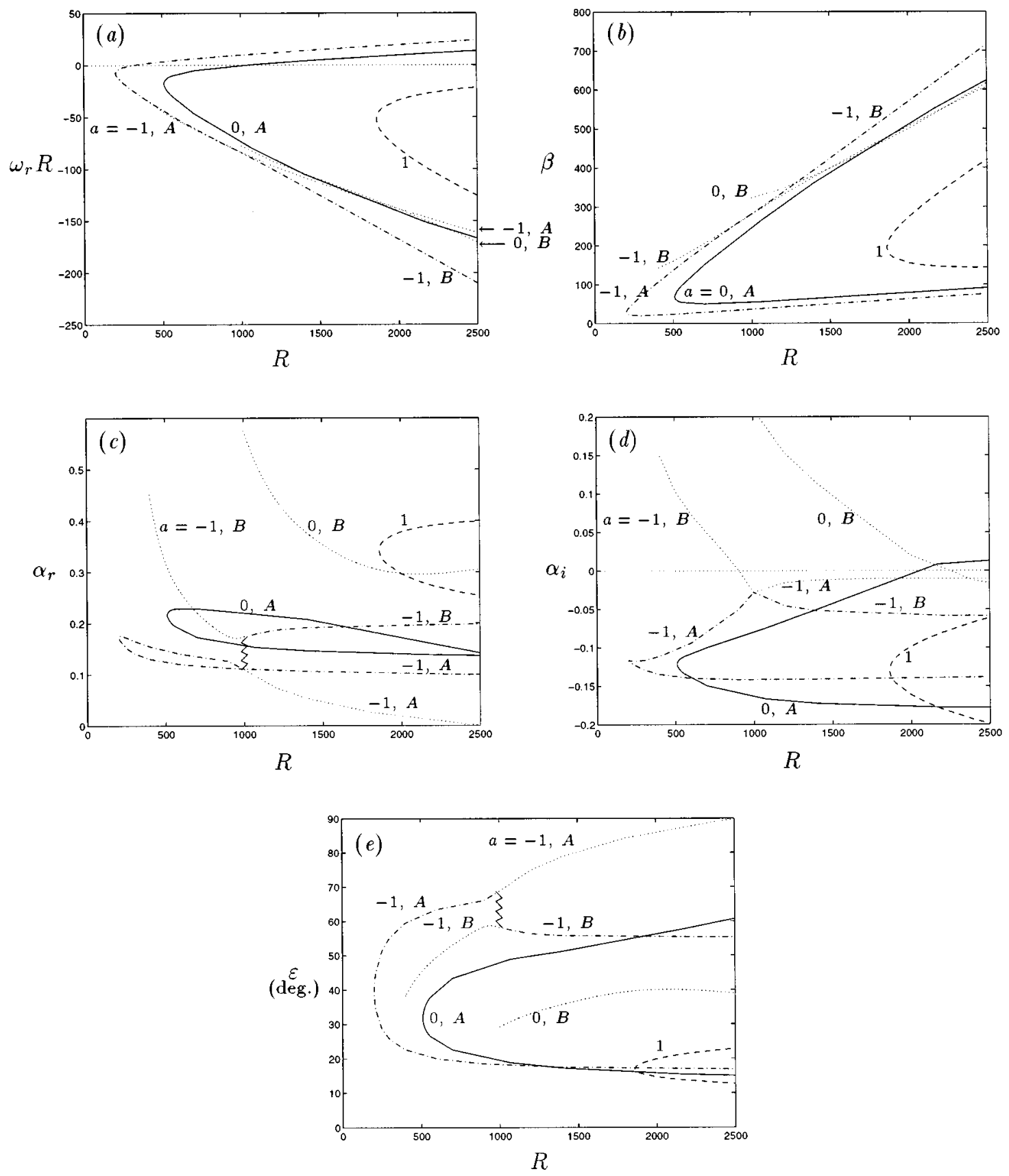

FIG. 6. Marginal curves for absolute instability: $-\cdots-$ denotes $a=-1$, - denotes $a=0$ and --- denotes $a=1$. A second trajectory of neutral $\left(\omega_{i}=0\right)$ branch points is given for $a=0$ and $a=-1$, which includes both non-pinching and pinching branch points. The marginal curves switch from trajectory $A$ to trajectory $B$ (marked by a zig-zag line) if the trajectory- $B$ branch points are of the pinch type and are more critical than those of trajectory $A$. Non-limiting trajectories are denoted by $\cdots$.

ingly close to the critical Reynolds number for the onset of absolute instability. Figure 9 and Table III summarize the effect of mass transfer on the onset of absolute instability (denoted by the subscript $c$ ). These critical values were calculated at intervals of 0.1 in $a$, over the range $-1 \leqslant a \leqslant 1$, but Table III gives just a small selection of these values.

The eigenfunctions at the critical parameters for the onset of absolute instability for $a=-0.5,0,0.5$ are given in Fig. 10. Compared with typical (travelling) branch-1 and branch-2 eigenfunctions, which are shown in Fig. 5, these eigenfunctions are not strikingly different. The noticeable differences are that the higher maxima in $\hat{u}$ are less pronounced here and the eigenfunctions of $\hat{v}$ have a small second maximum. As for the branch-1 and branch-2 eigenfunctions, injection increases the relative magnitudes of $\hat{u}$ and $\hat{w}$ compared with $\hat{v}$; suction decreases them.

\section{DISCUSSION}

Comparison between the absolute instability predicted by linear stability analysis and experimental observations for 


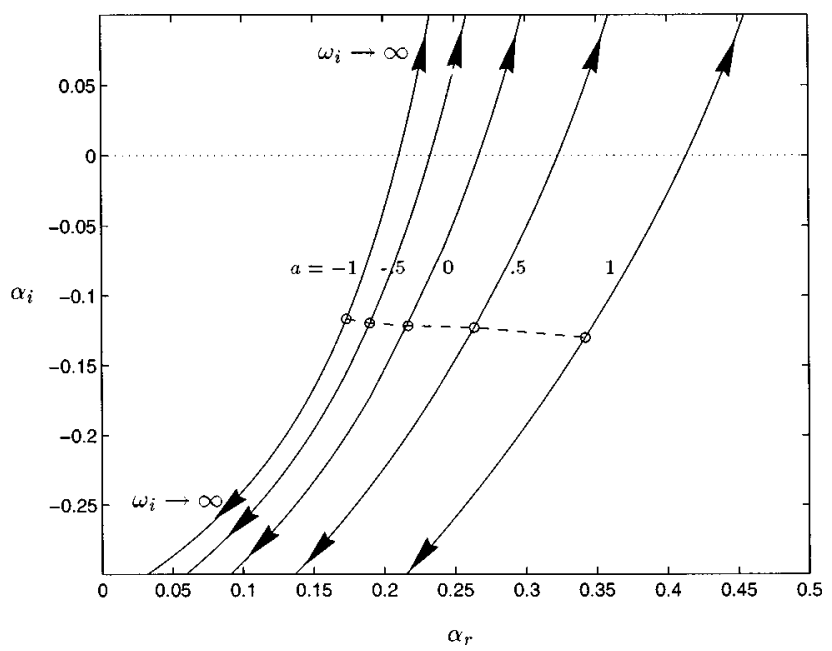

FIG. 7. Separation of the two spatial branches that coalesce at each branch point as $\omega_{i} \rightarrow \infty$ away from the values at the branch points $(\bigcirc)$. For each value of $a$, the critical pinch point for the onset of absolute instability is shown.

the zero-suction case are given by Lingwood. ${ }^{6,7}$ The onset of absolute instability was found to occur at $R \approx 510$ (corrected in Lingwood ${ }^{29}$ to about 507) and the onset of transition is consistently found by various experimentalists (see Lingwood $^{7}$ for a summary) at an average value of 513 with only a 3\% scatter around this value. It has been suggested that the onset of absolute instability promotes the onset of non-linearity and laminar-turbulent transition.

The effect of suction on the stability of the branch-1 crossflow vortices and transition of the rotating-disk boundary layer was studied experimentally by Gregory and

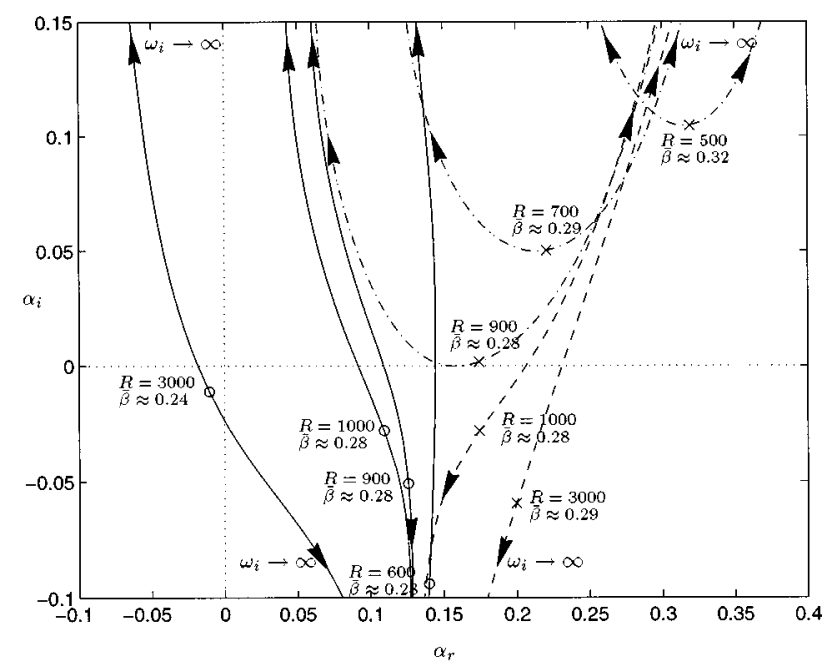

FIG. 8. Separation of the two spatial branches that coalesce at each branch point, as $\omega_{i} \rightarrow \infty$ away from the values at the branch points, evaluated at a selection of points on the branch-point trajectories shown in Fig. 6. Pinch points are indicated by $\longrightarrow$ and --- , and $-\cdot-$. indicates nonpinching branch points, Trajectory- $A$ branch points $(\bigcirc)$ are denoted by - , while trajectory- $B$ branch points $(\times)$ are denoted by --- and $-\cdot-\cdot$
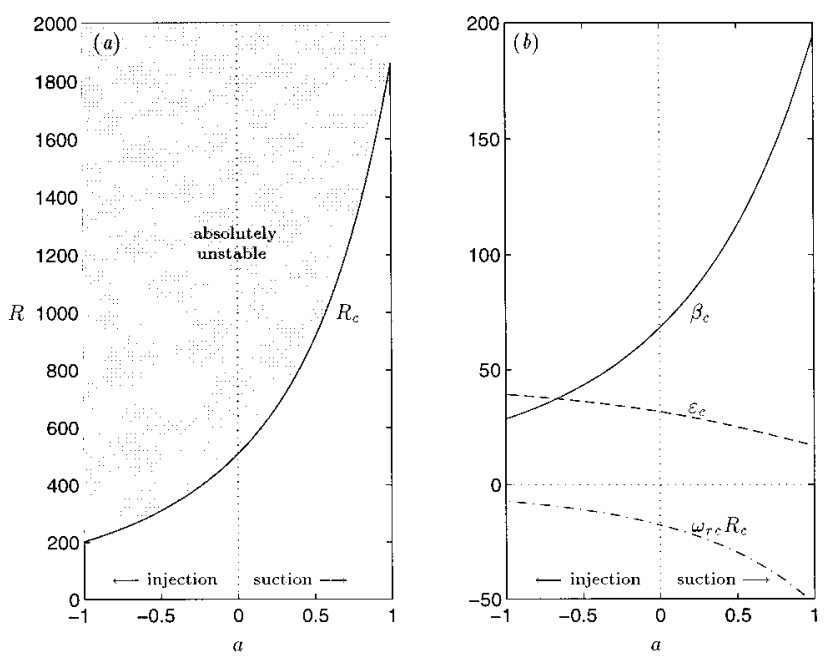

FIG. 9. (a) Variation in the critical Reynolds number for the onset of absolute instability with $a$, where the shaded region indicates the absolutely unstable region. (b) Variation in the critical azimuthal wavenumber (-), wave angle $(---)$ and frequency $(-\cdot-\cdot)$ for the onset of absolute instability with $a$.

Walker. ${ }^{18}$ They found, using a microphone probe and a hotfilm anemometer, that suction through both distributed suction holes and through slots increased the Reynolds number for instability and for transition above the values for zero suction. The distributed suction was achieved through a woven wire-cloth surface, but the critical Reynolds numbers of observed effects were dependent on the rotational speed of the disk because of the significant roughness of the disk. For the flow with suction slots they found that effects were less dependent on rotational speed and that the Reynolds number for the onset of transition increased from about 524 for no suction to about 632 with $a=0.4$. For the slotted disk, it was found that about $75 \%$ more suction was required to achieve a given level of stabilization than was predicted by stability theory, i.e., predicted by the increase in the critical Reynolds number for convective instability of branch- 1 crossflow vortices with suction. This discrepancy was attributed to limitations in the experimental apparatus, such as significant nonuniformity in suction (both radially and circumferentially), roughness of the disk surface and surface waviness. Here, it has been found that the onset of absolute instability occurs at $R \approx 803$ for a truly uniform suction of $a=0.4$. The Reynolds number for the onset of transition observed by Gregory and Walker ${ }^{18}$ for this suction rate is about $79 \%$ of this Reynolds number, but the absolute instability mechanism could still be

TABLE III. Critical values for the onset of absolute instability.

\begin{tabular}{rcccc}
\hline \hline \multicolumn{1}{c}{$a$} & \multicolumn{1}{c}{$R_{c}$} & $\bar{\beta}_{c}$ & $\omega_{c}$ & $\alpha_{c}$ \\
\hline-1.0 & 201.54 & 0.1424 & -0.03622 & $0.1735-i 0.1166$ \\
-0.5 & 309.71 & 0.1398 & -0.03550 & $0.1903-i 0.1197$ \\
0.0 & 507.30 & 0.1348 & -0.03485 & $0.2173-i 0.1216$ \\
0.5 & 911.54 & 0.1235 & -0.03261 & $0.2637-i 0.1228$ \\
1.0 & 1860.82 & 0.1044 & -0.02796 & $0.3420-i 0.1300$ \\
\hline \hline
\end{tabular}




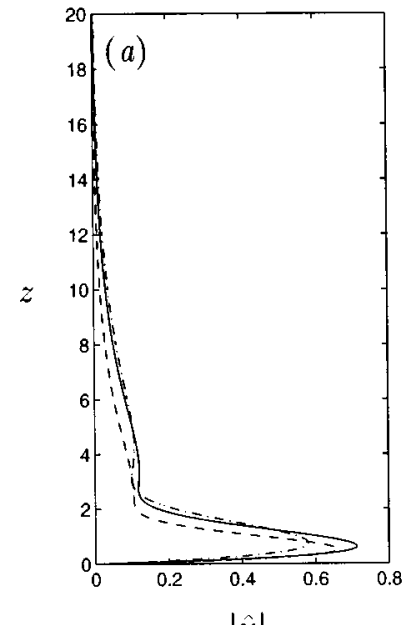

$|\hat{u}|$
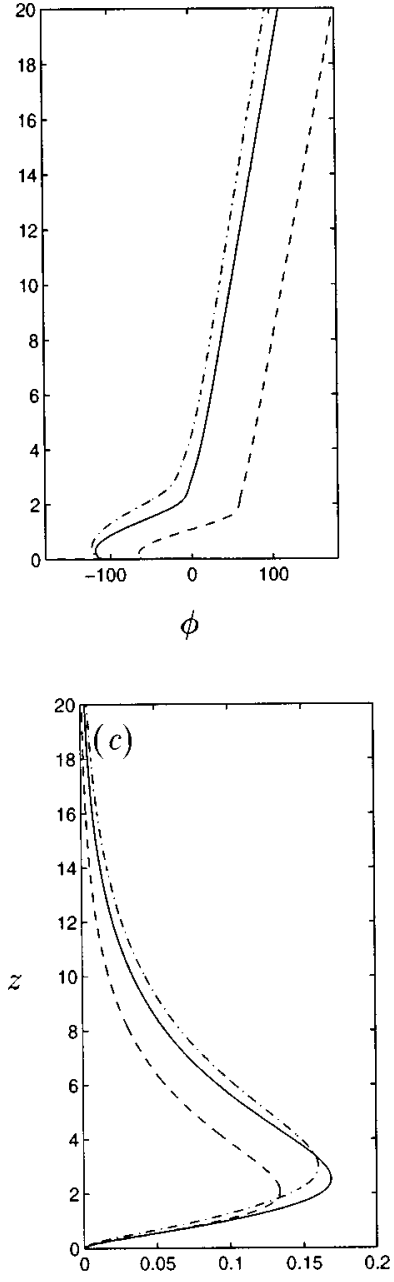

$|\hat{w}|$
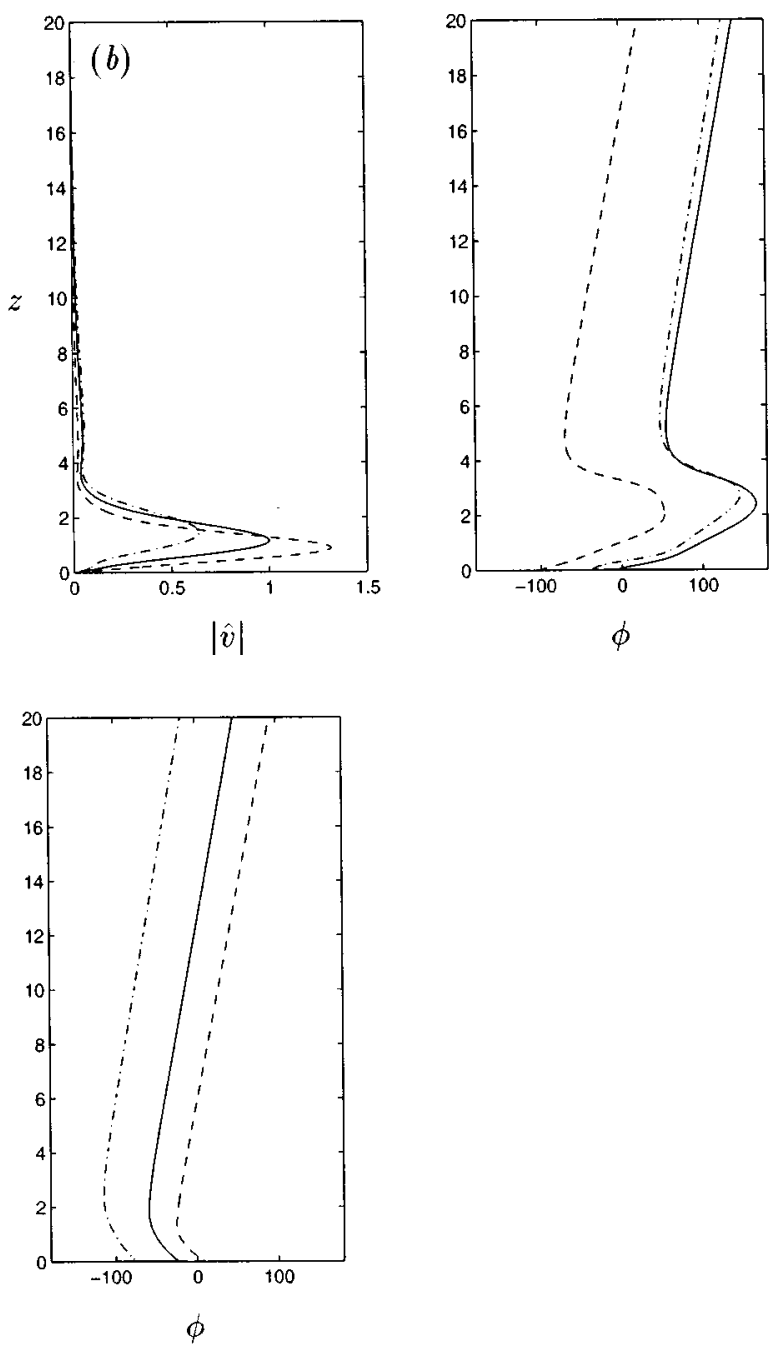

FIG. 10. Magnitude and phase $\phi$ of the eigenfunctions, normalized by the maximum of $\hat{v}$ for $a=0$, at the critical parameters for the onset of absolute instability: (a) radial perturbation velocity, (b) circumferential perturbation velocity, (c) axial perturbation velocity. The phase is given from $-180^{\circ}$ to $180^{\circ}$ and $-\cdot-$ denotes $a=-0.5$, denotes $a=0$ and --- denotes $a=0.5$.

responsible for the onset of transition if the limitations of the apparatus also reduced the effectiveness of suction to stabilize the absolute instability.

Gregory and Walker ${ }^{18}$ found that the maximum stabilizing effect (with the slotted surface) was produced for a relatively low suction rate, beyond which the onset of transition dropped back to a minimum (still at higher Reynolds number than the zero-suction case) and then increased again with increasing suction. With higher rates of suction it was suggested that the reduction in the mean radial velocity component allowed turbulent contamination to spread inwards from the rim. That is, the radial propagation somewhere within the wedge of disturbances excited by fixed roughnesses within the turbulent regime was zero, allowing the spread of disturbances both inwards and outwards of the roughness as the disturbances convected predominantly in the azimuthal direction. Gregory and Walker ${ }^{18}$ called this effect "selfcontamination," but is equally well described as radial absolute instability. This self-contamination was observed at Reynolds numbers below those predicted here for the onset of absolute instability, but this again may be attributable to reduced effectiveness of the experimentally-achieved suction compared with that of uniform suction.

It is worth noting that the methods used by Gregory and Walker ${ }^{18}$ to induce suction seemed to introduce significant roughness to the surface of the disk. Three-dimensional boundary layers, such as the rotating-disk boundary layer, are particularly sensitive to surface roughness. Furthermore, the non-linear study by Bassom and Seddougui ${ }^{17}$ suggests that the rotating-disk boundary layer with suction is even more sensitive than the zero-suction case, because suction reduces the threshold amplitude for subcritical growth of stationary disturbances. To properly assess the importance of the absolute instability as a transition mechanism in the rotating-disk boundary layer with mass transfer, it would be necessary to perform specific experiments with this in mind. For example, to make comparisons between the results from the linear stability analysis, which uses mean profiles calculated on the assumption of uniform mass transfer, it would be necessary to limit the surface roughness introduced by the 
suction holes, to achieve mass transfer as uniformly as possible and to consider both travelling and stationary disturbances within the boundary layer. It is, however, quite possible, particularly if the disk is rough, that the convective instability of stationary crossflow vortices (perhaps causing secondary instability) is the dominant transition mechanism.

\section{CONCLUSIONS}

The theoretical behaviour of the laminar rotating-disk boundary-layer flow with mass transfer at the wall has been investigated using linear stability theory. The effects of mass transfer on the convective instability of stationary modes have been studied previously (see, for example, Dhanak ${ }^{16}$ ). This investigation has been focused on the mass-transfer effects on the radial absolute instability, which has been shown to exist above a critical Reynolds number for the flow without mass transfer (see Lingwood ${ }^{7}$ ), and also on the effects of mass transfer on the convective instability of travelling modes. It has been shown that suction has a stabilizing effect on the absolute instability, while injection has a destabilizing effect. For increasing injection rates, the stationary waves become absolutely unstable at Reynolds numbers approaching the critical Reynolds number for the onset of absolute instability. The stationary waves are of particular importance because they are excited by unavoidable roughnesses on the surface of the disk, and are therefore often observed in experiments as stationary crossflow vortices.

Given sufficient time, an absolutely unstable flow will cause a disturbance at fixed points in space (in this case, at fixed radii) to grow to amplitudes large enough to make the use of the linear theory invalid. A disturbance in a convectively unstable flow, however, is swept away as it grows, and so the boundary layer remains basically laminar until the instability wave has travelled far enough away to have grown to amplitudes sufficient to cause non-linearities. Thus, absolute instability is quite distinct from, and more "dangerous" than, spatial instability. The absolute instability mechanism may explain the onset of non-linearity and laminar-turbulent transition at a well-defined radial position. Furthermore, the presence of an absolute instability would imply that any asymptotic stability analysis should be temporal as well as spatial.

The parallel-flow approximation will have some small numerical effect on the stability calculations, but it is expected that the general absolute instability characteristics discussed in this paper are still relevant to the physical behaviour of the flows; as has been shown for the particular case of zero mass transfer (Lingwood ${ }^{6,7}$ ) where the onset of transition and absolute instability coincide. Furthermore, for the case with zero mass transfer, the absolute instability mechanism has been shown to persist in the inviscid limit [where all terms $O\left(R^{-1}\right)$ are neglected consistently; see Lingwood $^{7}$ ], which indicates that the absolute instability is not an artifact of the parallel-flow approximation.

For all the flows, the absolute instability is caused by a pinch point between a spatially growing and a spatially damped branch of the dispersion relation. Apart from the special case where the pinch point lies on the real wavenumber axis, this must always be the case because in order for a branch point to be of the pinch type the two spatial branches that coalesce must originate in separate halves of the complex wavenumber plane for large positive imaginary frequency. To pinch, one of these branches must cross the real wavenumber axis, becoming convectively (spatially) unstable before pinching. So, the absolute instability always involves a branch of the dispersion relation that from a convective viewpoint is damped, and which is generally ignored for this reason.

In the convectively unstable regime, suction is strongly stabilizing to both stationary and travelling inviscidly unstable branch-1 modes; injection is destabilizing. Stationary viscously unstable branch-2 modes are strongly stabilized and destabilized by suction and injection, respectively, but travelling branch-2 modes are much less sensitive to mass transfer through the disk.

\section{ACKNOWLEDGMENTS}

This work was performed while supported by a Research Fellowship at Pembroke College, Cambridge.

${ }^{1}$ W. E. Gray, "The nature of the boundary layer at the nose of a swept back wing," unpublished, Min. Aviation, London, 1952.

${ }^{2}$ N. Gregory, J. T. Stuart, and W. S. Walker, "On the stability of threedimensional boundary layers with application to the flow due to a rotating disk," Philos. Trans. R. Soc. London Ser. A 248, 155 (1955).

${ }^{3}$ M. R. Malik, "The neutral curve for stationary disturbances in rotatingdisk flow," J. Fluid Mech. 164, 275 (1986).

${ }^{4} \mathrm{P}$. Hall, "An asymptotic investigation of the stationary modes of the instability of the boundary layer on a rotating disc," Proc. R. Soc. London Ser. A 406, 93 (1986).

${ }^{5}$ B. I. Fedorov, G. Z. Plavnik, I. V. Prokhorov, and L. G. Zhukhovitskii, "Transitional flow conditions on a rotating disc," J. Eng. Phys. 31, 1448 (1976).

${ }^{6}$ R. J. Lingwood, "An experimental study of absolute instability of the rotating-disk boundary-layer flow,', J. Fluid Mech. 314, 373 (1996).

${ }^{7}$ R. J. Lingwood, "Absolute instability of the boundary layer on a rotating disk," J. Fluid Mech. 299, 17 (1995).

${ }^{8}$ Y. Kohama, 'Study on boundary layer transition of a rotating disk," Acta Mech. 50, 193 (1984).

${ }^{9}$ Y. Kohama, "Crossflow instability in rotating disk boundary layer," AIAA Paper No. 87-1340, 1987.

${ }^{10}$ S. Balachandar, C. L. Streett, and M. R. Malik, "Secondary instability in rotating disk flow," AIAA Paper No. 90-1527, 1990.

${ }^{11}$ Th. von Kármán, “Über laminare und turbulente Reibung,"' Z. Angew. Math. Mech. 1, 233 (1921).

${ }^{12} \mathrm{G}$. K. Batchelor, "Note on the class of solutions of the Navier-Stokes equations representing steady non-rotationally symmetric flow," Q. J. Mech. Appl. Math. 4, 29 (1951).

${ }^{13}$ W. Pfenninger and J. W. Bacon, “Amplified laminar boundary layer oscillations and transition at the front attachment line of $45^{\circ}$ swept flat-nosed wing with and without boundary layer suction," in Viscous Drag Reduction, edited by C. S. Wells (Plenum, New York, 1969), p. 85.

${ }^{14}$ P. Hall, M. R. Malik, and D. I. A. Poll, "On the stability of an infinite swept attachment line boundary layer,' Proc. R. Soc. London Ser. A 395, 229 (1984).

${ }^{15}$ J. T. Stuart, "On the effects of uniform suction on the steady flow due to a rotating disk,"' Q. J. Mech. Appl. Math. 7, 446 (1954).

${ }^{16}$ M. R. Dhanak, "Effects of uniform suction on the stability of flow on a rotating disc," Proc. R. Soc. London Ser. A 439, 431 (1992).

${ }^{17}$ A. P. Bassom and S. O. Seddougui, "The effects of suction on the nonlinear stability of the three-dimensional boundary layer above a rotating disc,"' Proc. R. Soc. London Ser. A 436, 405 (1992).

${ }^{18} \mathrm{~N}$. Gregory and W. S. Walker, "Experiments on the effects of suction on the flow due to a rotating disk," J. Fluid Mech. 9, 225 (1960)

${ }^{19}$ S. O. Seddougui and A. P. Bassom, "The effects of suction on the nonlinear stability of a three-dimensional compressible boundary layer,' IMA J. Appl. Math. 56, 183 (1996). 
${ }^{20} \mathrm{P}$. Huerre and P. A. Monkewitz, "Local and global instabilities in spatially developing flows," Annu. Rev. Fluid Mech. 22, 473 (1990).

${ }^{21}$ R. J. Briggs, in Electron-Stream Interaction with Plasmas (MIT Press, Cambridge, 1965), Chap. 2.

${ }^{22}$ A. Bers, "Linear waves and instabilities," in Physique des Plasmas, edited by C. DeWitt and J. Peyraud (Gordon \& Breach, New York, 1975), p. 117.

${ }^{23} \mathrm{H}$. Schlichting, in Boundary-Layer Theory, 7th ed. (McGraw-Hill, New York, 1979), p. 102.

${ }^{24}$ M. R. Malik, S. P. Wilkinson, and S. A. Orszag, "Instability and transition in rotating disk flow," AIAA J. 19, 1131 (1981).
${ }^{25}$ R. J. Lingwood, "On the application of the Briggs' and steepest-descent methods to a boundary-layer flow," Stud. Appl. Math. (in press).

${ }^{26} \mathrm{~W}$. Koch, “Direct resonance in Orr-Sommerfeld problems,"' Acta Mech. 58, 11 (1986).

${ }^{27}$ D. S. Henningson and P. J. Schmid, "Vector eigenfunction expansions for plane channel flows," Stud. Appl. Math. 87, 15 (1992).

${ }^{28} \mathrm{P}$. Balakumar and M. R. Malik, "Traveling disturbances in rotating-disk flow," Theor. Comput. Fluid Dyn. 2, 125 (1990).

${ }^{29}$ R. J. Lingwood, "Absolute instability of the Ekman layer and related rotating flows," J. Fluid Mech. 331, 405 (1997) 